\title{
STRATEGIC IRRATIONALITY IN EXTENSIVE GAMES
}

\author{
Kaushik BASU* \\ Delhi School of Economics, Delhi, 110007, India \\ Communicated by A. Sen \\ Received 2 June 1986 \\ Revised 17 July 1987
}

\begin{abstract}
Most standard solution concepts of extensive games are history-insensitive in the sense that no matter at which information set one is, it is assumed that each player believes that everybody else is rational. In reality, each history of moves reveals certain traits of the players to one another. It is argued in this paper that solution concepts ought to make use of this fact. An example of such a concept is developed and called a 'reasonable solution' set. It is shown that this can explain cooperation in certain finitely-repeated games like the Prisoner's Dilemma.
\end{abstract}

Key words: Extensive games; rationality; cooperation; Prisoner's Dilemma.

\section{Introduction}

Most solution concepts of extensive games, e.g., perfection (Selten, 1975) or rationalizability (Pearce, 1984) are 'history-insensitive', in the sense that no matter at which information set one is in the game, in what remains it is implicitly assumed that each player believes that everybody else is rational. That is, even if a player has revealed himself irrational, others continue to believe he is rational. In reality, a particular history of moves may reveal to a player traits of the other players and thereby influence his play in the remainder of the game. The present paper is an attempt to introduce this idea formally.

Another motivation for this paper is the inability of existing solution concepts to explain cooperation among players in some repeated games. The most prominent example is the finitely-repeated Prisoner's Dilemma where common sense suggests that there may be cooperation in the early games but standard solutions, including rationalizability, predict noncooperation throughout. In an important contribution, Kreps et al. (1982) have explained cooperation in early games by assuming that one player believes that the other person may be a tit-for-tat player. This is however not enough because in reality even when two experts in game theory, who have no $a$ priori doubts about each other's expertise, play the Prisoner's Dilemma we expect

\footnotetext{
* I am grateful to Pradeep Dubey, Vijay Krishna, Arunava Sen, Kunal Sengupta and Nirvikar Singh for comments.
} 
to see some cooperation. How does one explain this? While no firm answer is given here, I will argue that an important step to explaining this is to look for a suitable history-sensitive solution concept. The specific one suggested in this paper is intended to motivate such a search.

Attention is confined here to games of perfect information. This has the virtue of simplicity; but, more importantly, in games of perfect information the set of possible outcomes according to different solution criteria tend to coincide. Thus though the formal model here is developed along the lines of Pearce, my critique is applicable to the perfect equilibrium concept as well.

I motivate the reader by presenting a game which shares the basic problem of repeated Prisoner's Dilemma but has the advantage of even greater simplicity. The game may be called passing-the-parcel: There are two players, One and Two, and One has a parcel with him. The first move is One's. He can keep the parcel (fink) or pass it on to Two (coop). If he finks he gets three units of money and Two gets nothing and the game ends there. If he coops, each player gets two units of money and it is now player Two's move. Player Two may keep it, in which case he gets three units and the other player nothing and the game ends there, or he may pass it on which gives each player two units. And so on. The hundreth move is the last one: suppose starting from the first one, coop has been played 99 times. Now the parcel is with player Two. If he plays fink, he gets three units and One gets zero and the game ends. If he plays coop, they both get two units and the game ends. The total amount that a player earns is the sum of his earnings after each move as specified above.

What is the expected outcome of this game? Note first that if both players coop throughout, each player gets a total of 200 . However, all standard solution concepts predict a unique outcome: Player One will fink in the first move and that will be the end with player One having earned three and player Two, zero! The argument is the familiar backward induction one (see, e.g., Kreps et al., 1982). The trouble with this solution is that it is experimentally invalidated and seems flawed on introspection.

To explain the essential shortcoming of rationalizability or perfection in explaining the outcome of passing-the-parcel, it is enough to consider a 3-move variant of the 100-move version just described. The game is illustrated on the next page. 


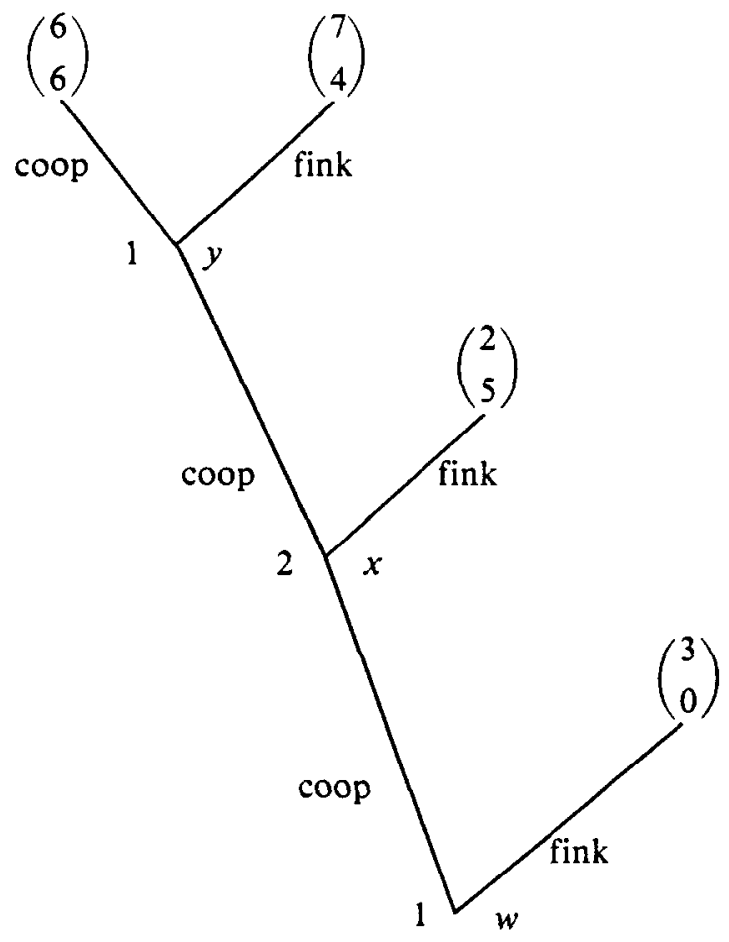

Since this is a game of perfect information we need not distinguish between a node and an information set. The backward induction argument goes as follows: At node $y$, One will fink and get 7 instead of cooping and getting 6 . Since Two knows that One is rational, he knows this. So at node $x$, Two will fink rather than coop. Since finking gives him 5 and cooping 4. Since One knows that Two is rational and that Two knows that One is rational, at node $w$, One will fink!

Let us examine this last step carefully. If One finks, het gets 3 . If instead he coops, the game moves on to node $x$. Now consider Two pondering his decision at node $x$. If he believes that One is rational, he should fink. But, at $x$, should he believe One is rational? It is not clear that he should. The fact that the game has reached $x$ means One is not rational in a conventional sense. Two will be quite baffled and it will not be unreasonable if he supposes that One is an 'unpredictable' player and may play coop at $y$. So he, in turn, may move coop at $x$. And realizing this, One may find it worthwhile to move coop at the initial node $w$. The argument is much stronger in the early play of longer games.

Playing coop at $w$ would be irrational by the conventional standards of game theory. But it is an 'irrational' move made for strategic reasons. Also interesting to note is that Two's move coop at node $x$ is not irrational. Because in the light of One 
having revealed his irrationality, playing coop at $x$ can no longer be ruled out. Of course, at $y$, One will always play fink, since One's earlier 'irrational' move was merely meant to confuse Two, and did not reflect actual irrationality.

The direction we will explore has the shortcoming that generally no unique prediction is possible. But the response to this is similar to the one given by Pearce (1984): That in many extensive game situations a wide variety of outcomes are plausible. To rule these out by some rule of thumb does not enhance our predictive ability. Also, the spirit of the argument that 1 will use is very similar to that of Bernheim and Pearce. It gives us a broadened solution concept which is really the outcome of pursuing their own argument further.

Having done this, we may once again undertake research to refine our solution. One may think of some routes of solution-refinement undertaken in the literature; e.g., Myerson (1978) or Pearce (1984) in his Section 5. This has not been done in this paper which merely suggests a way of building in history-sensitivity and thereby of understanding why cooperation may occur in the early games in repeated game theory or why individuals may make moves which appear irrational.

\section{Basic concepts}

An extensive game of perfect information is described briefly, following mainly the notation of Kreps and Wilson (1982) and Mclennan (1985).

An extensive game, $\Gamma$, consists of a finite set, $T$, of nodes, which is strictly partially ordered by $<$. For all $x, y \in T$, ' $x<y$ ' represents ' $x$ precedes $y$ ' or ' $y$ succeeds $x$ '. The pair $(T,<)$ forms an arborescence. ${ }^{\prime}$ An initial node is one with no predecessor. $\Gamma$ has a unique initial node and the symbol $w$ is reserved for it. For any node $x \in T$, its immediate predecessor (if it exists) is denoted by $p_{1}(x)$. The $n$th predecessor of $x$ is defined inductively as follows: $p_{n}(x)=p_{1}\left(p_{n-1}(x)\right)$. For all $x \in T$, we define $p_{0}(x)=x$. An immediate successor of $x$ is defined analogously. For any $x \in T, I$ shall use $S(x)$ to denote the set of immediate successors of $x$. The set of terminal nodes is denoted by $Z=\{z \in T \mid S(z)=\phi\}$ and the set of nonterminal nodes, also known as decision nodes, is denoted by $H=T \backslash Z$.

Also specified in the game, $\Gamma$, is a finite set, $A$, of actions, and a mapping $\alpha$,

$$
\alpha: T \backslash\{w\} \rightarrow A,
$$

where $\alpha(x)$ is the last action taken to reach $x$. For all $x \in H, \alpha(S(x))$ is the set of actions available at $x$. This is denoted, in brief, as $A(x)$. That is, $A(x)=\alpha(S(x))$. I assume $\{A(x) \mid x \in H\}$ is a partition of $A$.

Next we have a set $I=\{1, \ldots, N\}$ of players, and $\left\{H^{i}\right\}_{i \in I}$ is a partition of $H$. If $x \in H^{i}$, then player $i$ has to choose an action at $x$. Since $\Gamma$ is a game of perfect information there is no need to distinguish between nodes and information sets.

\footnotetext{
${ }^{1}$ That is, for all $x \in T$, the set of predecessors of $x$ is completely ordered by $<$.
} 
Finally, specified in $\Gamma$ is a utility function for each player $i$ :

$$
u^{i}: Z \rightarrow R, \quad \forall i \in I,
$$

where $R$ is the set of real numbers.

For all $x \in H$, if we consider $x$ and all the successors of $x$ in $T$ and take the restriction of all the above definitions to this subset of $T$, then this comprises a game which has $x$ as its initial node. Such a game is called a subgame of $\Gamma$ and is denoted by $\Gamma_{x}$. For a more precise definition of subgames, see Kreps and Wilson (1982).

A pure strategy of player $i$ is a mapping

$$
s^{i}: H^{i} \rightarrow A
$$

such that for all $x \in H^{i}, s^{i}(x) \in A(x)$. We denote the set of all pure strategies of player $i$ by $S^{i}$. For any finite set $F$, let $\Delta(F)$ be the set of all probability distributions over $F$ :

$$
\Delta(F) \equiv\left\{m \in R^{F} \mid \sum_{t \in F} m(t)=1, m(t) \geq 0, \forall t \in F\right\} .
$$

The set of all mixed strategies of player $i$ is given by $\Delta\left(S^{i}\right)$. A mixed strategy $m \in \Delta\left(S^{i}\right)$ which gives a probability of one to $s \in S^{i}$ is not distinguished here from $s$.

For any subgame $\Gamma_{x}$, I use $S_{x}^{i}$ to denote the set of all pure strategies of player $i$ in $\Gamma_{x}$.

Given sets $T^{1}, \ldots, T^{N}$, I shall denote its Cartesian product, $T^{1} \times \cdots \times T^{\vee}$, by $\Pi T^{i}$ or $\Pi \Pi T^{r}$. Let $s=\left(s^{1}, \ldots, s^{\nu}\right) \in \Pi S^{i}$ and $x \in T$. We say that $x$ is reached by $s$ if the following is true: Either $x=w$ or $w$ is the $n$th predecessor of $x$ and with $i(t)$ denoting the player whose move it is at $p_{t}(x)$, we have $s^{i(t)}\left(p_{t}(x)\right)=\alpha\left(p_{t-1}(x)\right)$, for all $t \in\{1, \ldots, n\}$. If $s^{i} \in S^{i}$ and $x \in T$, we say that $s^{i}$ reaches $x$ if there exist $s^{j} \in S^{j}$, for all $j \neq i$ such that $\left(s^{1}, \ldots, s^{N}\right)$ reaches $x$.

Let $m=\left(m^{i}, \ldots, m^{N}\right) \in \Pi \Delta\left(S^{i}\right)$ and $x \in T$. We say that $x$ is reached by $m$ if there exists $\left(s^{1}, \ldots, s^{N}\right)$ such that $m^{i}\left(s^{i}\right)>0$, for all $i \in I$ and $\left(s^{1}, \ldots, s^{N}\right)$ reaches $x$. The probability of reaching $x$, given that $m$ is being played, is denoted by $p(x, m)$ :

$$
\sum_{s \text { reaches } x} m^{1}\left(s^{1}\right) m^{2}\left(s^{2}\right) \cdots m^{N}\left(s^{N}\right)=p(x, m) .
$$

The expected utility function of $i, U^{i}: \Pi \Delta\left(S^{i}\right) \rightarrow R$, is derived in a usual manner from $i$ 's utility function. That is, for all $m \in \Pi \Delta\left(s^{i}\right)$,

$$
U^{i}(m)=\sum_{z \in Z} u^{i}(z) p(z, m)
$$

Given a strategy $N$-tuple $m \in \Pi \Delta\left(S^{r}\right)$ and a strategy $\beta^{i} \in \Delta\left(S^{i}\right), m / \beta^{i}$ is the strategy $N$-tuple derived by replacing the $i$ th-element of $m$ with $\beta^{i}$. Let $s^{i} \in B^{i} \subset S^{i}$. Then $s^{i}$ is a best response to $m \in \Pi \Delta\left(S^{r}\right)$ among all elements of $B^{i}$ (or, simply, in $B^{i}$ ) if

$$
U^{i}\left(m / s^{i}\right) \geq U^{i}\left(m / t^{i}\right), \quad \forall t^{i} \in B^{i} .
$$

Given $s \in S^{i}$ and $x \in H$, an $x$-replacement of $s$ is any strategy $t \in S^{i}$ such that for 
all $y \in H$ such that $y \neq x$ and $y$ is not a successor of $x, s(y)=t(y)$.

For a player $i$ playing $s \in S^{i}$ a conjecture is a mapping,

$$
c_{s}^{i}: H^{i} \rightarrow \Delta\left(S^{1}\right) \times \cdots \times \Delta\left(S^{i-1}\right) \times\{s\} \times \Delta\left(S^{i+1}\right) \times \cdots \times \Delta\left(S^{*}\right),
$$

such that for all $x \in H^{i}$ that can be reached by $s, c_{s}^{i}(x)$ reaches $x$, and if $x<y$ and $c_{s}^{i}(x)$ reaches $y$ then $c_{s}^{i}(x)=c_{s}^{i}(y)$.

By saying that $i$ playing $s$ has a conjecture, we mean that at each node which can be reached by $s$ and where $i$ has to choose an action, $i$ has a belief about each $j \neq i$ as to which pure strategy he may be playing with some probability. This is equivalent to conjecturing that $j$ is playing a particular mixed strategy (Pearce, 1984, Appendix A). It will be foolish of him if he believes people are playing strategies which cannot reach the node that has been reached. So we rule this out. Also, till a player's belief about what the others are playing is actually revealed to be false, he does not change his belief.

Now we define for each player his set of 'rationalizable strategies'. Informally speaking, a player is rational if his chosen strategy can be thought of as a best response to some conjecture at each decision node of this player. It is assumed that players are rational, they know that they are rational, etc. In short, the rationality of players is 'common knowledge'. Hence, each player will first delete his strategies which are not best responses to some strategy vector which the others might adopt. Having done this, and knowing that others have also done the same, each player will once again check whether further strategies can be deleted. Such iterative deletion continues and what remains in the end comprises the set of rationalizable strategies. Now for the formal definition.

Given $X^{r} \subset \Delta\left(S^{r}\right)$, for all $r \in I$, and $s \in S^{i}$, define $J^{i}\left(s,\left\{X^{r}\right\}\right)=\left\{x \in H^{i} \mid \exists m \in \Pi X^{r}\right.$, such that $m / s$ reaches $x\}$. Thus $J^{i}\left(s,\left\{X^{r}\right\}\right)$ is the set of nodes in $H^{i}$ which can be reached if $i$ plays $s$ and for all $r \in I \backslash\{i\}$, he expects $r$ to choose a strategy from $X^{r}$.

Definition 1. For all non-negative integer $t$, and for all $i \in I$, the set $S^{i}(t)$ is defined iteratively as follows:

For all $i \in I, S^{i}(0)=S^{i}$.

$s \in S^{i}(t)$ iff $s \in S^{i}(t-1)$ and $\mathrm{B}$ a conjecture $c_{s}^{i}$ such that for all $x \in J^{i}\left(s,\left\{S^{r}(t-1)\right\}\right), c_{s}^{i}(x) \in \Delta\left(S_{1}(t-1)\right) \times \cdots \times \Delta\left(S^{N}(t-1)\right)$; and $s$ is a best response to $c_{s}^{i}(x)$ among all $x$-replacements for $s$ in $S^{i}(t-1)$.

The set of rationalizable strategies of player $i$ in $\Gamma$ is denoted by $R^{i}(\Gamma)$, and is defined as follows.

$$
R^{i}(\Gamma)=\bigcap_{t=0}^{\infty} S^{i}(t), \quad i \in I .
$$

The collection of all rationalizable strategy $N$-tuples $\Pi R^{i}(\Gamma)$ is labelled the rationalizable solution set of $\Gamma$. 


\section{Strategic irrationality}

A shortcoming of this solution concept is its history-insensitivity. Let $\Gamma_{x}$ be a subgame of $\Gamma$. The rationalizable solution set of $\Gamma_{x}$ in no way depends on the information revealed in the fact of node $x$ having been reached. That is, if there is another subgame $\Gamma_{y}$, identical to $\Gamma_{x}$, then its solution set must be identical to that of $\Gamma_{x}$. Players learn nothing by observing how others have played thus far, while contemplating moves! But surely an essential feature of an extensive game is that players have the scope for learning about others during the course of the game. What I proceed to do is to introduce such learning in a small way.

First note that in the model of Section 2, it is implicitly built in that each player believes everybody is rational and he maintains this belief no matter where he is in the game tree.

Let us see what the solution set of $\Gamma$ would look like if a subset, $\Omega$, of the players is believed to be irrational. It is not being assumed that members of $\Omega$ are irrational, but simply that for each $i \in \Omega$, all $j \in I \backslash\{i\}$ believe that $i$ is irrational and it is common knowledge that all $j \in I \backslash\{i\}$ believe that $i$ is irrational. If a player is irrational - we shall often refer to him as unpredictable - it means that he may choose any available strategy. A game $\Gamma$ in which $\Omega$ is the set of players believed to be unpredictable will be denoted by $(\Gamma, \Omega)$. The rationalisable strategies of players for a game $(\Gamma, \Omega)$ may now be formally defined.

Definition 2. For all non-negative integer, $t$, and for all $i \in I$, we define a set $\bar{S}^{i}(t) \subset S^{i}$ as follows: $\forall i \in \Omega$ and $\forall t$, and $\bar{S}^{i}(t)=S^{i}$. And $\forall i \notin \Omega, \bar{S}^{i}(0)=S^{i}$ and $\bar{S}^{i}(t)$ is defined recursively as follows: $s \in \bar{S}^{i}(t)$ iff $s \in \bar{S}^{i}(t-1)$ and $\exists$ a conjecture $c_{s}^{i}$ such that $\forall x \in J^{i}\left(s,\left\{S^{r}(t-1)\right\}\right)$,

$c_{s}^{i}(x) \in \Delta\left(\bar{S}^{1}(t-1)\right) \times \cdots \times \Delta\left(S^{N}(t-1)\right)$; and $s$ is a best response to $c_{s}^{i}(x)$ among all $x$-replacements for $s$ in $\bar{S}^{i}(t-1)$.

For all $i \in I$, define $\bar{R}^{i}(\Gamma, \Omega)=\bigcap_{t=0}^{\infty} \bar{S}^{i}(t)$.

The set of rationalizable strategies of player $i$ in $(\Gamma, \Omega)$ is denoted by $R^{i}(\Gamma, \Omega)$ and is defined as follows:

$$
\begin{aligned}
& \forall i \in I \backslash \Omega, R^{i}(\Gamma, \Omega)=\bar{R}^{i}(\Gamma, \Omega), \text { and } \\
& \forall i \in \Omega, s \in R^{i}(\Gamma, \Omega) \text { iff } s \in S^{i} \text { and }
\end{aligned}
$$

$\exists$ a conjecture $c_{s}^{i}$ such that $\forall x \in J^{i}\left(s,\left\{R^{r}(\Gamma, \Omega)\right\}\right), c_{s}^{i}(x) \in \Delta\left(\bar{R}^{1}(\Gamma, \Omega) \times \cdots \times\right.$ $\Delta\left(R^{N}(\Gamma, \Omega)\right)$; and $s$ is a best response to $c_{s}^{i}(x)$ among all $x$-replacements of $s$. The rationalizable solution set for $(\Gamma, \Omega)$ is $\Pi R^{i}(\Gamma, \Omega)$.

It is important to understand why for $i \in \Omega, R^{i}(\Gamma, \Omega) \neq \bar{R}^{i}(\Gamma, \Omega)$. Since such an $i$ is believed to be irrational, others believe he may play anything in $\bar{R}^{i}(\Gamma, \Omega)$ which 
is equal to $S^{i}$. But since $i$ is actually rational, he would not consider playing any strategy in $S^{i}$ but only those which are best responses to what he believes others may play. $R^{i}(\Gamma, \Omega)$ is the set of such moves.

Note that once we have Definition 2, Definition 1 becomes a special case where for all $i \in I, R^{i}(\Gamma)=R^{i}(\Gamma, \phi)$.

The next step involves identifying unpredictable players. Since each node $x \in T$, has a history of moves, at each such node we could think of a set of players who appear as unpredictable to others. We begin by formalizing this idea in an abstract form. Let $2^{I}$ be the power set of $I$. The function, $\Omega$, defined as

$$
\Omega: H \rightarrow 2^{l}
$$

will be called a predictability map. A predictability map identifies at each node the set of players who appear unpredictable or irrational to others.

In this paper I shall be concerned with a specific predictability map, one suggested naturally by the works of Bernheim and Pearce. I shall assume that a player is believed to be rational till he is revealed to be not so, in the sense of Pearce (1984), that is, till he makes a move that is not rationalizable. Hence $\Omega(w)=\phi$. Now consider a node $y$, where $\Omega(y)=\phi$, and $i$ has to move. Suppose he chooses an action which is incompatible with any rationalizable strategy of his and which takes the game to node $x$. Then it is reasonable to suppose that, $\Omega(x)=\{i\}$. Hence in the subgame $\Gamma_{x}$, we would expect each player $j$ to adopt a strategy belonging to $R^{j}\left(\Gamma_{x},\{i\}\right)$.

Similarly if $\Omega(x)$ is the set of players believed to be unpredictable at node $x$, then $x$ onwards we expect a player $j$ to play from $R^{j}\left(\Gamma_{x}, \Omega(x)\right)$. If $j$ makes a move incompatible with any strategy in this set his name gets added to the set of players believed to be unpredictable. This informal idea is now made rigorous.

Definition 3. A specific predictability map, $\bar{\Omega}$, is defined as follows. Let $x \in T$ and $p_{n}(x)=w$. Let $\tau:\{1, \ldots, n\} \rightarrow I$ be such that $\forall j, \tau(j)$ satisfies $p_{j}(x) \in H^{\tau(j)}$. If $x$ can be reached by some $s \in \Pi R^{r}(r, \phi)$, then $\bar{\Omega}(x)=\phi$. If not, then let $p_{t_{1}}(x)$ be the closest predecessor of $x$ reached by some $s \in \Pi R^{r}(\Gamma, \phi)$. If $x$ can be reached by some $s \in \Pi R^{r}\left(\Gamma_{p_{1}(x)},\left\{\tau\left(t_{1}\right)\right\}\right)$, then $\bar{\Omega}(x)=\left\{\tau\left(t_{1}\right)\right\}$. If not, then let $p_{t_{2}}(x)$ be the closest predecessor of $x$ that can be reached by some $s \in \Pi R^{r}\left(\Gamma_{p_{1}(x)},\left\{\tau\left(t_{1}\right)\right\}\right)$. If $x$ can be reached by some $s \in \Pi R^{r}\left(\Gamma_{p_{t}(x)},\left\{\tau\left(t_{1}\right), \tau\left(t_{2}\right)\right\}\right)$, then $\Omega(x)=\left\{\tau\left(t_{1}\right), \tau\left(t_{2}\right)\right\}$. If not, we continue in the same way. Since this is a finite game, we must reach $t_{k}$ such that $\left\{\tau\left(t_{1}\right), \ldots, \tau\left(t_{k}\right)\right\}=\bar{\Omega}(x)$.

In Pearce's work, the predictability map used implicitly is one where $\Omega(x)=\phi$, for all $x$. Once this is amended, as per Definition 3, it becomes necessary to redefine rationalizable strategies. We do this by defining sophisticated rationalizable strategies of each player.

Definition 4. For all $J \in 2^{l}$, define $K(J)=\{x \in H \mid \bar{\Omega}(x)=J\}$. Let $n(J)$ be the number of nodes in $K(J)$ with no predecessors in $K(J)$. Label these nodes 
$x_{1}(J), \ldots, x_{n(J)}(J)$. If $K_{j}(J)$ is a set containing $x_{j}(J)$ and all its successors in $K(J)$, then $\left\{K_{1}(J), \ldots, K_{n(J)}(J)\right\}$ is a partition of $K(J)$. The set of sophisticated rationalizable strategies of player $i$ in $\Gamma$ is denoted by $E^{i}(\Gamma)$ and defined as follows:

$$
\begin{aligned}
E^{i}(\Gamma)=\left\{s \in S^{i} \mid \forall J, \forall j, \text { if } K_{j}(J) \cap H^{i} \neq \phi,\right. \text { then } \\
\left.\qquad \exists t \in R^{i}\left(\Gamma_{x_{j}(J)}, J\right) \text { such that } \forall x \in K_{j}(J) \cap H^{i}, \quad s(x)=t(x)\right\} .
\end{aligned}
$$

A person playing a strategy in $E^{i}(\Gamma)$ is just a more sophisticated version of Pearce's player. What he does is this. He begins by assuming that everybody is rational, chooses a rationalizable strategy and continues to play it, as long as his initial assumption of everybody being rational is not contradicted. As soon as he reaches a node where somebody has been revealed irrational, he revises his initial opinion and computes the set of his rationalizable strategies taking into account that $\Omega$ is no longer empty. He continues with a rationalizable strategy in this subgame till he once again reaches a node where some other player is revealed irrational. And so on. $^{2}$

This approach has the weakness that unless someone is revealed irrational, others believe he will continue to make rationalizable moves only. Irrationality is never anticipated. Nevertheless, this is an improvement over standard approaches where even after a player is revealed to be irrational, others believe he is rational. It is therefore claimed that for purposes of prediction we ought to, as a first step, eschew rationalizability in favor of sophisticated rationalizability. However, as the next theorem suggests, this will not make any difference in terms of outcomes.

For any set $G \subset \Pi S^{i}$, define $\theta(G)=\{z \in Z \mid z$ can be reached by some $s \in G\} . \theta(G)$ consists of terminal nodes which can be reached by some strategy $N$-tuple in $G$. If $G$ and $G^{\prime}$ are such that $\theta(G)=\theta\left(G^{\prime}\right)$, we say that $G$ and $G^{\prime}$ are realization equivalent.

Theorem 1. Given any game $\Gamma$, (i) the rationalizable solution set of $\Gamma$ and $\Pi E^{i}(\Gamma)$ are realization equivalent, and (ii) $E^{i}(\Gamma)$ is non-empty, $\forall i \in I$.

Proof. (i) Let $z \in \theta\left(\Pi R^{i}(\Gamma)\right)$ and $s=\left(s^{1}, \ldots, s^{N}\right) \in \Pi R^{i}(\Gamma)$ be such that $s$ reaches $z$. Hence $\bar{\Omega}(z)=\phi$ and $\forall x<z, \bar{\Omega}(x)=\phi$.

$\forall i \in I$, construct $t^{i} \in S^{i}$ as follows: $\forall J \in 2^{\prime}$ and $\forall j \in\{1, \ldots, n(J)\}$ such that $K_{j}(J) \cap H^{i}=\phi$, choose any strategy from $R^{i}\left(\Gamma_{x_{j}(J)}, J\right)$ and call it $r^{i j J}$. Note that $R^{i}\left(\Gamma_{x_{j}(J)}, J\right)$ must be non-empty since $R^{i}\left(\Gamma_{x_{j}(J)}, \phi\right)=R^{i}\left(\Gamma_{x_{j}(J)}\right)$ is a subset of it and the non-emptiness of $R^{i}\left(\Gamma_{x_{j}(J)}\right)$ is assured by proposition 4 in Pearce (1984). Now,

\footnotetext{
${ }^{2}$ When it is said that player $j$ has been revealed irrational, it means that he has been observed making a move which is not rationalizable. It is not necessary that others actually think that $j$ is irrational. They may well be aware that $j$ is up to some tricks. But if others reason as players do in the papers of Bernheim and Pearce, they will be puzzled by $j$ 's behavior. And even if they realize that clever $j$ is up to some tricks, they will not be able to figure out what the tricks are. It is simply being assumed that under such circumstances they will treat $j$ as an unpredictable player.
} 
$\forall x \in K_{j}(J) \cap H^{i}$, define $t^{i}(x)=r^{i j J}(x)$. Since $\left\{K_{j}(J) \cap H^{i}\right\}_{j, J}$ is a partition of $H^{i}$, this completely defines $t^{i}(x)$, and by the mode of construction it follows that $t^{i} \in E^{i}(\Gamma)$. Since $x<z$ implies $x \in K(\phi)$, if $x<z$ and it is player $\tau(x)$ 's move at $x$, then $t^{\tau(x)}(x)=$ $s^{\tau(x)}(x)$. Hence, $\left(t^{1}, \ldots, t^{v}\right)$ reaches $z$. Thus $z \in \theta\left(\Pi E^{i}(\Gamma)\right)$.

To prove the converse suppose $z \in \theta\left(\Pi E^{i}(\Gamma)\right)$ and $s=\left(s^{1}, \ldots, s^{N}\right) \in \Pi E^{i}(\Gamma)$ reaches $z$. We shall prove by induction that $\forall x<z, \bar{\Omega}(x)=\phi$. By definition $\bar{\Omega}(w)=$ $\phi$. Suppose $x$ is such that $w<x<z$ and $\bar{\Omega}\left(p_{1}(x)\right)=\phi$. Let $p_{1}(x)$ be $i$ 's decision node. Then $\exists t^{i} \in R^{i}\left(\Gamma_{w}, \bar{\Omega}\left(w^{\prime}\right)\right)=R^{i}(\Gamma)$ such that $s^{i}\left(p_{1}(x)\right)=t^{i}\left(p_{1}(x)\right)$. Hence $\bar{\Omega}(x)=\phi$.

Let $M^{i}=\left\{x \in H^{i} \mid x<z\right\}$. If $s^{i} \in E^{i}(\Gamma)$, then $\exists t^{i} \in R^{i}\left(\Gamma_{w}, \bar{\Omega}(w)\right)=R^{i}(\Gamma)$ such that $\forall x \in M^{i}, s^{i}(x)=t^{i}(x)$. For each $i \in I$, choose such a $t^{i}$. Hence $t=\left(t^{1}, \ldots, t^{N}\right) \in \Pi R^{i}(\Gamma)$ and $t$ reaches $z$. This completes the proof of (i).

(ii) Proposition 4 of Pearce (1984) ensures the non-emptiness of the rationalizable solution set. Hence (i) implies (ii).

In playing a sophisticated rationalizable strategy, a player takes into account whether someone has revealed himself as irrational. But since no one makes an "irrational' move, nodes where $\Omega \neq \phi$ are never reached. This is the idea behind part (i) of Theorem 1.

However, once we realize that players will play sophisticated rationalizable strategies, we cannot really rule out strategic irrational moves. Players may have an advantage in appearing irrational. The main solution concept suggested in this paper, allows for such strategic irrationality. We define this next.

Definition 5. The set of reasonable strategies of player $i$ in $\Gamma$, denoted by $D^{i}(\Gamma)$ is defined as follows: $s \in D^{i}(\Gamma)$ iff $\exists$ a conjecture $c_{s}^{i}$ such that $\forall x \in J^{i}\left(s,\left\{E^{r}(\Gamma)\right\}\right)$, $c_{s}^{i} \in \Delta E^{1}(\Gamma) \times \cdots \times \Delta E^{i-1}(\Gamma) \times\{s\} \times \Delta E^{i+1}(\Gamma) \times \cdots \times \Delta E^{N}(\Gamma)$ and $s$ is a best response to $c_{s}^{i}(x)$ among all $x$-replacements for $s$ in $S^{i}$. The reasonable solution set of $\Gamma$ is $\Pi D^{i}(\Gamma)$.

Theorem 2. The reasonable solution set of a game, $\Gamma$, is non-empty.

Proof. By Theorem $1, E^{r}(I) \neq \phi, \forall r \in I$. Let $m^{1}, \ldots, m^{N}$ be such that $\forall j \neq i, m^{j}$ is a mixed strategy giving positive weights to all strategies in $E^{j}(\Gamma)$. Since $S^{i}$ is finite, $\exists s \in S^{i}$ such that $s$ is a best response to $m^{1}, \ldots, m^{N}$ in $S^{i}$.

Now define a mapping

$$
c_{s}^{i}: H^{i} \rightarrow \Delta\left(S^{1}\right) \times \cdots \times \Delta\left(S^{i-1}\right) \times\{s\} \times \Delta\left(S^{i-1}\right) \times \cdots \times \Delta\left(S^{N}\right)
$$

such that $c_{s}^{i}(x)=\left(m^{1}, \ldots, m^{i-1}, s, m^{i+1}, \ldots, m^{N}\right), \forall x \in H^{i}$. It is easy to check that $c_{s}^{i}$ is a conjecture for player $i$ playing $s$, and that $\forall x \in J^{i}\left(s,\left\{E^{r}(\Gamma)\right\}\right), s$ is a best response to $c_{s}^{i}(x)$ among all $x$-replacements of $s$. Hence $s \in D^{i}(\Gamma)$. 


\section{Examples}

The most interesting f zature of a reasonable solution is that it allows for cooperation in games like passing-the-parcel discussed in Section 1. In that example, one reasonable solution is where where One plays coop at $w$ and Two plays fink at $x$. The reason why One may play coop at $w$ is that this being incompatible with his rationalizable strategy he will appear irrational at $x$, i.e., $\Omega(x)=\{1\}$. That being so, Two may play fink or coop (the latter with the conjecture that One may, thanks to his unpredictability, play coop at $y$ ). Hence a possible conjecture of One is that, if $x$ is reached, Two will play coop. And in that case One's best response is to play coop at $w$.

A slight variation of the same game could make the reasonable strategy set of each player unique. Consider the game $\Gamma^{\prime}$.

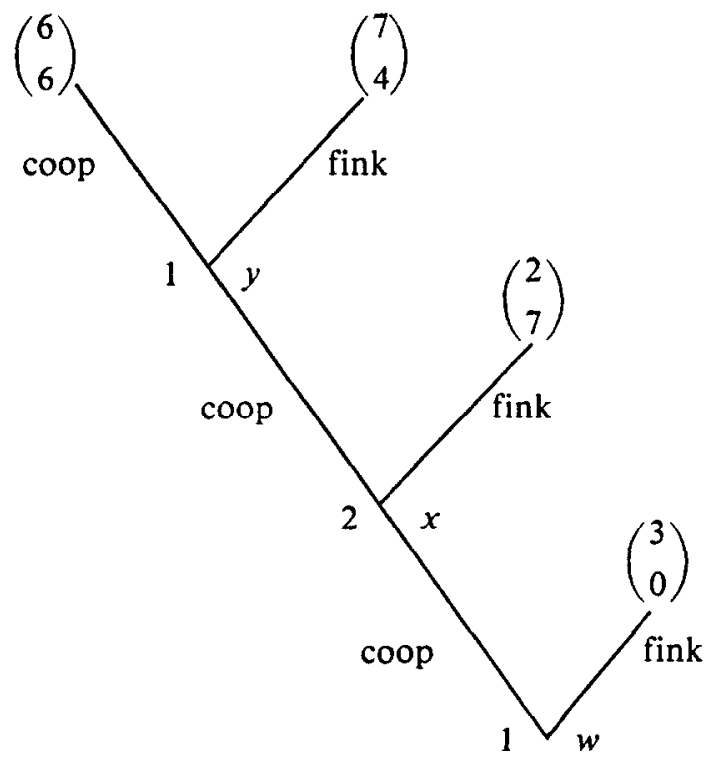

$\Gamma^{1}$

In $\Gamma^{1}$, One has nothing to gain by appearing irrational, since at $x$, Two will necessarily play fink. Hence One's only reasonable strategy is to fink at $w$.

Consider the next game $\Gamma^{2}$, which has been discussed by Pearce. If player Two is reached, clearly he plays $\beta_{1}$. Knowing this and knowing that he cannot influence Two's play by appearing to be irrational, One will play $\alpha_{2}$. Hence, the reasonable solution is unique and it coincides with Pearce's rationalizable solution. Since in $\Gamma^{2}$, One playing $\alpha_{1}$ and Two playing $\beta_{2}$ is a Nash equilibrium, this shows that not all Nash equilibria are reasonable. The above examples illustrate that in noncontroversial cases the reasonable and rationalizable outcomes coincide. Difference of opinion arises in more controversial games and this paper argues that in such cases, the criterion of reasonableness is the more appealing. 


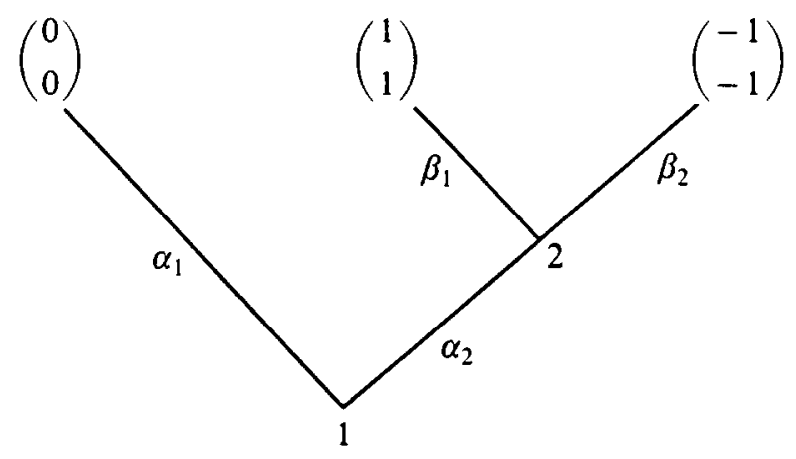

$\Gamma^{2}$

Given a game $\Gamma$, we say that this is an $n$-period game if $n=\max _{z \in Z} 1(z)$, where $1(z)$ is the number of nodes preceding $z$. From the above examples it should be clear that for all games of less than three periods, the rationalizable outcomes and reasonable outcomes coincide. This is because, in such games no player can influence others by appearing to be irrational. This means that in games like passing-theparcel cooperation can be explained only if the games are played over sufficient periods, namely three or more. As a future research project one may try to include more structure in the reasonableness criterion so that we obtain results like - the longer the game, the more likely is cooperation in the early games.

The kind of argument applicable to passing-the-parcel carries over, by analogy, to the finitely-repeated Prisoner's Dilemma. ${ }^{3}$ It may be worthwhile for a player to adopt the cooperative strategy in the first game and thereby appear unpredictable to the opponent. This breaks down the backward induction argument. Once this happens, it is entirely possible for the two players to play coop for some time though they are bound to end up noncooperating.

\section{Conclusion}

Though in this paper attention was confined to games of perfect information, it is not difficult to see that similar arguments hold for games of imperfect information. Detailed analysis will have to await the formalization of reasonableness for imperfect-information games. But, in the meantime, some intuitive remarks may be instructive.

The main difficulty with a formal definition of reasonableness for games of imperfect-information is that there may be no obvious way of defining a predictability map. That is, there is no obvious unique counterpart of Definition 3. First of all, note that $\Omega$ will be defined for each information set instead of node. Now there may exist an information set which can be reached if either one of two players has

\footnotetext{
${ }^{3}$ And much of the same argument carries over to the Chain Store Paradox (Selten, 1978) as well. It is, however, interesting to note that in a game where the opponent changes with each repetition cooperation is unlikely.
} 
been irrational. Which player should the decision-maker at this information set take to be unpredictable? This is the kind of conceptual issue that one would need to solve before developing a formal framework.

There are however games where such conceptual problems do not arise and one can informally isolate reasonableness in imperfect-information games using the argument developed in this paper. Consider Kohlberg's celebrated example, reproduced here as $\Gamma^{3}$.

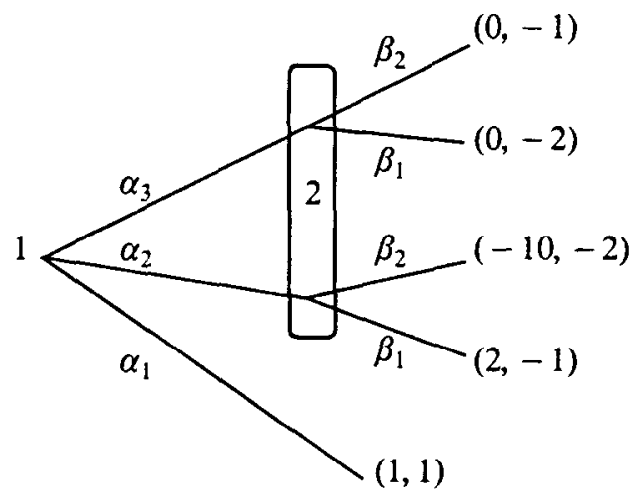

Since $\Gamma^{3}$ is a two-period game no player has an advantage in appearing irrational. Hence, the same argument as in Pearce (p. 1044) applies here and the only reasonable strategy is $\alpha_{2}$ for player One and $\beta_{1}$ for player Two. Since One playing $\alpha_{1}$, and Two playing $\beta_{2}$ is a perfect equilibrium and also a sequential equilibrium, this shows that not all perfect or sequential equilibria are reasonable.

In the traditional analysis of an extensive game of perfect information if a player makes a move which is not rationalizable, other players ignore this information. This paper tries to take into account the fact that players are sensitive to such information and may make use of it. There are, however, alternative ways in which a player may interpret the irrationalizable move of another player. Consequently by adopting alternative assumptions we could modify the criterion of reasonable strategies developed in this paper. Just to take an example suppose that we assume that if any player $j$ makes a move (at a node that is not terminal or preterminal) which is not rationalizable then others believe that $j$ is rational but does not know that others (i.e., all $i \neq j$ ) are rational. This would give us a slightly modified criterion of reasonableness which would imply for games like the repeated Prisoner's Dilemma a slightly earlier breakdown of cooperation.

These are alternatives worth exploring but the essential point stressed here is that as an extensive game proceeds, players may modify their opinion about one another's rationality. This, in turn, means that some player may try to influence the opinions of others regarding his rationality. To ignore this is to ignore an essential informational aspect of the extensive form. ${ }^{4}$

\footnotetext{
${ }^{4}$ Around the time that this paper was being written, Phil Reny was working on the same theme and reached similar conclusions (see Reny, 1986), as we discovered later on meeting.
} 


\section{References}

D. Bernheim, Rationalizable strategic behavior, Econometrica 52 (1984) 1007-1028.

D. Kreps, P. Milgrom, J. Roberts and R. Wilson, Rational cooperation in the finitely-repeated Prisoner's Dilemma, J. Econ. Theory 27 (1982) 245-252.

D. Kreps and R. Wilson, Sequential equilibria, Econometrica 50 (1982) 863-894.

A. Mclennan, Justifiable beliefs in sequential equilibrium, Econometrica 53 (1985) 889-904.

R.B. Myerson, Refinements of the Nash equilibrium concept, Int. J. Game Theory 7 (1978) 73-80.

D.G. Pearce, Rationalizable strategic behavior and the problem of perfection, Econometrica 52 (1984) 1029-1050.

P. Reny, Rationality, Common knowledge and the theory of games, Ph.D. dissertation, Princeton University, 1986.

R. Selten, Reexamination of the perfectness concept for equilibrium points in extensive games, Int. J. of Game Theory 4 (1975) 25-55.

R. Selten, The chain store paradox, Theory and Decision 9 (1978) 127-159. 